\title{
The Dyadosphere of black holes and gamma-ray bursts
}

\section{R. Ruffini}

I.C.R.A.-International Center for Relativistic Astrophysics and Physics Department, University of Rome "La Sapienza", I-00185 Rome, Italy

e-mail: ruffini@icra.it

Received January 21; accepted March 12, 1999

Abstract. Works on the Dyadosphere are reviewed.

Key words: black holes physics — gamma-rays: bursts

I am proposing and give reasons that with Gamma Ray Bursts, for the first time we are witnessing, in real time, the moment of gravitational collapse to a Black Hole. Even more important, the tremendous energies involved by the energetics of these sources, especially after the discoveries of their afterglows and their cosmological distances (Kulkarni et al. 1998), clearly point to the necessity and give the opportunity to use as an energy source of these objects the extractable energy of Black Holes.

That Black Holes can only be characterized by their mass-energy $E$, charge $Q$ and angular momentum $L$ has been advanced in a classical article (Ruffini \& Wheeler 1971), the proof of this has been made after twenty five years of meticulous mathematical work. One of the crucial points in the Physics of Black Holes was to realize that energies comparable to their total mass-energy could be extracted from them. The computation of the first specific example of such an energy extraction process, by a experiment Gedanken, was given in (Ruffini \& Wheeler 1970) and (Floyd \& Penrose 1971) for the rotational energy extraction from a Kerr Black hole, see Fig. 1. The reason of showing this figure is not only to recall the first such explicit computation, but to emphasize how contrived and difficult such a mechanism can be: it can only work for very special parameters and should be in general associated to a reduction of the rest mass of the particle involved in the process. To slow down the rotation of a Black Hole and to increase its horizon by the accretion of counterrotating particles is almost trivial, but to extract the rotational energy from a Black Hole, namely to slow down the Black Hole and keep its surface area constant, is extremely difficult, as clearly pointed out also by the example in Fig. 1. The above experiment Gedanken, extended as well to electromagnetic interactions, became of paramount importance not for their direct astrophys-

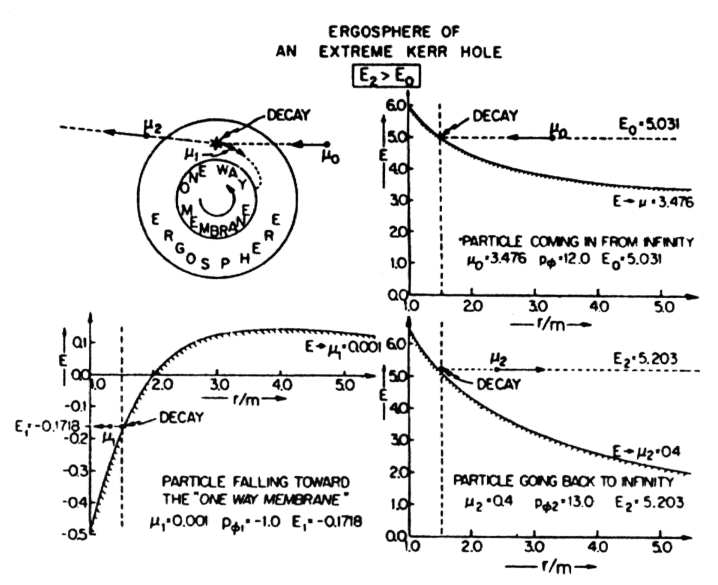

Fig. 1. (Reproduced from Ruffini and Wheeler with their kind permission.) Decay of a particle of rest-plus-kinetic energy $E_{\text {。 }}$ into a particle which is captured into the black hole with positive energy as judged locally, but negative energy $E_{1}$ as judged from infinity, together with a particle of rest-plus-kinetic energy $E_{2}>E_{\text {。 }}$ which escapes to infinity. The cross-hatched curves give the effective potential (gravitational plus centrifugal) defined by the solution $E$ of Eq. (2) for constant values of $p_{\phi}$ and $\mu$ (figure and caption reproduced from Christodoulou 1970)

ical significance but because they gave the tool for testing the physics of Black Holes and identifing their general mass-energy formula (Christodoulou \& Ruffini 1971). The crucial point is that a transformation at constant surface area of the Black Hole, or reversible in the sense of reference Christodoulou \& Ruffini (1971), could release an energy up to $29 \%$ of the mass-energy of an extremal rotating Black Hole and up to $50 \%$ of the mass-energy of an extremely magnetized and charged Black Hole.

Various models have been proposed in order to tap the rotational energy of Black Holes by the processes of relativistic magnetohydrodynamic. It is likely however that these processes are relevant over the very long time scales characteristic of the accretion processes. 
In the present case of the Gamma Ray Bursts a prompt mechanism, on time scales shorter than a second, for depositing the entire energy in the fireball at the moment of the triggering process of the burst, appears to be at work. For this reason we are here considering a more detailed study of the vacuum polarization processes a'la Heisenberg-Euler-Schwinger (Heisenberg \& Euler 1931; Schwinger 1951) around a Kerr-Newman Black Hole first introduced by Damour and Ruffini (Damour \& Ruffini 1975). The fundamental points of this process can be simply summarized:

- They occur in an extended region arround the Black Hole, the Dyadosphere, extending from the horizon radius $r_{+}$to the Dyadosphere radius $r_{\mathrm{ds}}$ see (Preparata et al. 1998a,b). Only Black Holes with a mass larger than the upper limit of a neutron star and up to a maximum mass of $610^{5} M_{\odot}$ can have a Dyadosphere, see (Preparata et al. 1998a,b) for details.

- The efficiency of transforming the mass-energy of Black Hole into particle-antiparticle pairs outside the horizon can approach 100\%, for Black Holes in the above mass range see (Preparata et al. 1998a,b) for details.

- The pair created are mainly positron-electron pairs and their number is much larger than the quantity $Q / e$ one would have naively expected on the ground of qualitative considerations. It is actually given by $N_{\text {pairs }}=\frac{Q}{e}\left(1+\frac{r_{\mathrm{ds}}}{\hbar / m c}\right)$, where $m$ is the electron mass. The energy of the pairs and consequently the emission of the associated electromagnetic radiation peaks in the $\mathrm{X}$-gamma rays region, as a function of the Black Hole mass.

I recall some of the results on the Dyadosphere. We consider the collapse to almost general Black Hole endowed with an electromagnetic field (EMBH). Following Preparata et al. (1998a,b), for simplicity we consider the case of a non rotating Reissner-Nordstrom EMBH to illustrate the basic gravitational-electrodynamical process. The number density of pairs created in the Dyadosphere is

$N_{\mathrm{e}^{+} \mathrm{e}^{-}} \simeq \frac{Q-Q_{\mathrm{c}}}{e}\left[1+\frac{\left(r_{\mathrm{ds}}-r_{+}\right)}{\frac{\hbar}{m c}}\right]$,

where

$r_{\mathrm{ds}}=\left(\frac{\hbar}{m c}\right)^{\frac{1}{2}}\left(\frac{G M}{c^{2}}\right)^{\frac{1}{2}}\left(\frac{m_{\mathrm{p}}}{m}\right)^{\frac{1}{2}}\left(\frac{e}{q_{\mathrm{p}}}\right)^{\frac{1}{2}}\left(\frac{Q}{\sqrt{G} M}\right)^{\frac{1}{2}}$.

Due to the very large pair density and to the sizes of the cross-sections for the process $\mathrm{e}^{+} \mathrm{e}^{-} \leftrightarrow \gamma+\gamma$, the system is expected to thermalize to a plasma configuration for which

$N_{\mathrm{e}^{+}}=N_{\mathrm{e}^{-}}=N_{\gamma}=N_{\text {pair }}$

and reach an average temperature

$k T_{\circ}=\frac{E_{\mathrm{e}^{+} \mathrm{e}^{-}}^{\text {tot }}}{3 N_{\text {pair }} \cdot 2.7}$, where $k$ is Boltzmann's constant. The discussions on the relativistic expansion of the Dyadosphere are presented in a separated paper (see e.g. Ruffini et al. 1999 in this proceeding).

Before concluding I would like to return to the suggestion, advanced by Damour and Ruffini, that a discharged EMBH can be still extremely interesting from an energetic point of view and responsible for the acceleration of ultrahigh energy cosmic rays. I would like just to formalize this point with a few equations: It is clear that no matter what the initial conditions leading to the formation of the EMBH are, the final outcome after the tremendous expulsion of the PEM pulse will be precisely a Kerr Newman solution with a critical value of the charge. If the background metric has a killing vector, the scalar product of the killing vector and the generalized momentum

$P_{\alpha}=m U_{\alpha}+e A_{\alpha}$,

is a constant along the trajectory of any charged gravitating particle following the relativistic equation of motion in the background metric and electromagnetic field (Jantzen \& Ruffini 1999). Consequently an electron (positron) starting at rest in the Dyadosphere will reach infinity with an energy $E_{\text {kinetic }} \sim 2 m c^{2}\left(\frac{G M}{c^{2}}\right) /\left(\frac{\hbar}{m c}\right) \sim 10^{22} \mathrm{eV}$ for $M=10 M_{\odot}$

\section{References}

Christodoulou D., Ruffini R., 1971, Phys. Rev. D 4, 3552

Damour T., Ruffini R., 1975, Phys. Rev. Lett. 35, 463

Heisenberg W., Euler H., 1931, Zeits. Phys. 69, 742

Floyd R.M., Penrose R., 1971, Nat 229, 177 (submitted) 16 December 1970

Jantzen R., Ruffini R. (to be submitted for publication 1999). This paper will also contain other relativistic considerations on the nature of the Dyadosphere

Kulkarni S.R., et al., 1998, Nat 395, 663 (for a complete set of reference see these proceedings)

Preparata G., Ruffini R., Xue S.-S., 1998a (submitted to Phys. Rev. Lett.); and 1998b, A\&A 338, L87

Ruffini R., Wheeler J.A., 1971, Introducing the Black Hole Physics Today 24, (1), 30

Ruffini R., Wheeler J.A., "Relativistic Cosmology from Space Platforms" in Proceedings of the Conference on Space Physics, Hardy V. and Moore H. (eds.). E.S.R.O. Paris, 1971. The preparation of this report took more than one year and the authors were unwilling to publish parts of it before the final publication. The manuscript was then incorporated in the book M. Rees, R. Ruffini and J.A. Wheeler "Black holes, Gravitational Waves and Cosmology" Gordon and Breach New York 1974 (also translated into Russian MIR 1973). In order to avoid these delays the results of the energy extraction process from a Kerr Black Hole, as well as the definition of the Ergosphere, were inserted as Fig. 1 in the Christodoulou 1970 paper (submitted 17 September 1970 and published 30 November 1970)

Ruffini R., Salmonson J.D., Wilson J.R., Xue S.S., 1999 (in these proceedings and references therein)

Schwinger J., 1951, Phys. Rev. D 82, 664 